\title{
The Interaction of Encoding and Rehearsal Processes in the Recall of Repeated and Nonrepeated Items ${ }^{1}$
}

\author{
DAVID G. ELMES \\ Washington and Lee University \\ AND \\ ROBERT A. BJORK \\ University of Michigan
}

\begin{abstract}
Experiments were designed to clarify the influence of level of processing on the recall of once-presented items and to determine whether spacing effects for repeated items result from differential processing of the second presentation. In a modified Brown-Peterson paradigm, the lengths of distractor-filled spacing and retention intervals were varied, and subjects were cued to rehearse each presentation of five-word pentads in either a primary (rote) or secondary (elaborative) fashion. Type of rehearsal had large and systematic effects, but the pattern of spacing effects was inconsistent with that predicted by a processing interpretation. An alternative theory, in which type of processing is assumed to interact with encoding variability, seems consistent with the results.
\end{abstract}

One of the most fundamental of human memory phenomena is that long-term recall of a repeated verbal item is an increasing function of the temporal spacing of the presentations of that item. The various theoretical efforts to account for such spacing effects (for reviews, see (Bjork, 1970; Hintzman, 1974; Melton, 1970)) have tended to assume that spacing increases either the amount of consolidation or rehearsal devoted to the item, or that spacing increases the independence of the encoding processes during the first and second presentations of the item and, hence, increases the average number of encodings. This paper reports on several experiments designed to test the idea that spacing

\footnotetext{
1 This research was conducted while the first author was a Visiting Research Associate at the Human Performance Center, Department of Psychology, University of Michigan for which support was provided by the Air Force Office of Scientific Research under Contract No. F44620-72-C-0038 and by the Advanced Research Projects Agency of the Department of Defense under Contract No. F44620-72-C-0019. We are grateful to Anne L. Elmes, Arthur M. Glenberg, and Robert H. Jongeward for their technical assistance. Copyright (C) 1975 by Academic Press, Inc. All rights of reproduction in any form reserved. Printed in Great Britain
}

effects result not from differences in amount of rehearsal or number of encodings but, rather, from different types of rehearsal or processing.

The major reason for suspecting that qualitative differences in rehearsal rather than sheer frequency of rehearsal may be implicated in the spacing effect is because a number of recent studies (Craik \& Watkins, 1973; Jacoby, 1973; Jacoby \& Bartz, 1972; Meunier, Ritz, \& Meunier, 1972; Woodward, Bjork, \& Jongeward, 1973) have demonstrated that in order to account for several other retention phenomena it is necessary to distinguish between rehearsal as a maintenance operation, primary rehearsal, and rehearsal as a constructive, elaborative process, secondary rehearsal. Primary rehearsal consists of a rote, cyclic repetition of a set of items; while in contrast, secondary rehearsal involves various mnemonic activities by means of which items within the set being rehearsed are associated or integrated. Increasing the amount of primary rehearsal has little, if any, effect on long-term recall; on the other hand, long-term recall 30 
benefits substantially from increases in the amount of secondary rehearsal.

The foregoing distinction between primary and secondary rehearsal suggests yet another possible explanation of the spacing effect: Massed repetitions tend to result in primary rehearsal of the second presentation, whereas spaced repetitions tend to yield secondary rehearsal of the second presentation. Thus, massed repetitions may not encourage subjects to "turn off the processor" as is assumed in the Underwood (1970) and Greeno (1970) formulations, but, rather, massed repetitions are seen as encouraging only rote cycling of the item at the time of its second presentation. When repetitions are massed or close in time, the item is remembered essentially without fail and with little effort from its first to its second presentation; therefore, from the subject's standpoint, nothing more seems required at the time of the second presentation than to recycle the item as encoded on the first presentation. In the case of spaced repetitions, however, the item is either not retrievable or is difficult to retrieve upon its second presentation; it may seem necessary, from the subject's standpoint, to encode the item again or to elaborate the initial encoding. Given such differences in processing the second presentation, spaced repetitions would, on the average, result in more encoding or more elaborated encoding than would massed repetitions, which, in turn, would produce better longterm recall.

It follows from the preceding levels-of-processing explanation of spacing effects that controlling the type of processing of the second presentation could eliminate the advantage of spaced over massed repetitions. In particular, if subjects are required to do primary processing of an item's second presentation, the spacing effect would be attenuated or eliminated because the minimal long-term benefits resulting from primary rehearsal should reduce the level of spaced-item recall to that of massed-item recall.

Requiring subjects to engage in secondary rehearsal of the second presentation may or may not yield a spacing effect. If the only mechanism responsible for the spacing effect is the level of processing of the second presentation, then there should be no spacing effect since level of processing is equated at all intervals. Regardless of the spacing interval, however, recall should be better than in the case where the subjects are instructed to process the second presentation in a primary fashion. If, on the other hand, one also assumes that the encoding variability principle is valid, then there should be a spacing effect when the repetition receives secondary rehearsal. The encoding variability principle, first formulated by Estes (1955a, b) and later modified and upheld by others (e.g. (DeRemer \& D'Agostino, 1974; Madigan, 1969; Martin, 1968; Melton, 1970)), states that the encoding of a verbal item at the time of a second presentation will overlap less with the encoding of the first presentation the greater the spacing or context change between the two presentations. Variable encoding, in turn, increases the number of available retrieval routes for an item and therefore enhances retention. Thus, in a situation where a massed and a spaced repetition are both rehearsed in a secondary fashion, one might still expect a spacing effect owing to more variable encoding for spaced repetitions than for massed ones. Any such spacing effect should be less than that obtained in an uninstructed control condition, however, if the level-of-processing and encoding variability notions are both valid.

The experiments reported below were designed to constitute a relatively straightforward test of these implications as well as to provide additional evidence concerning the role of types of processing in recall. In all three studies a modified Brown-Peterson paradigm was employed, and the subjects were required to rehearse and remember five-word strings in accord with a cue that indicated how to process the string at each presentation. The major point at issue is whether or not the spacing effect is a function of the type of rehearsal 
given to the second presentation. The three experiments reported below were designed to constitute a sequence that would converge on that issue. In the first experiment, type of rehearsal on each presentation of repeated items was varied orthogonally with spacing interval. In the second experiment, only those rehearsal conditions expected to have the greatest influence on the spacing effect were included, and an uninstructed control group was included to provide a base line against which to measure the instructed conditions. In the third experiment, the influence of the processing type at the time of the second presentation was examined in more detail by limiting the processing of the first presentation to one vocal repetition in all conditions. In all three experiments, subjects were given some trials on which the items were presented just once; those trials were included both to ascertain the effects of rehearsal mode on the recall of once-presented items and to try to insure that the subjects would engage in the appropriate type of rehearsal on the first presentation of a repeated item.

\section{EXPERIMENTS I AND II}

Experiments I and II were quite closely related. In both studies, the subjects were required, on each of a series of Brown-Peterson trials, to remember pentads comprised of five common four-letter nouns. The pentads were presented once or twice and most subjects were cued how to process the item on each presentation (i.e., they were cued to rehearse in either a primary or secondary fashion). At the end of each distractor-filled retention interval there was a recall test for the five words presented on that trial, and at the end of each experiment there were recall and recognition tests for all the words presented during the experiment.

\section{Method: Experiment I}

Subjects. Sixteen undergraduates at the University of Michigan served as paid subjects.
They were paid $\$ 2.00$ for their participation. All subjects had previously served in at least one memory experiment, but none of them had participated in a study that involved different types of rehearsal.

Apparatus and materials. The sequence of events on any one trial was shown in the window of a high-speed memory drum (change time less than $.01 \mathrm{sec}$ ). Advances of the memory drum were controlled by a papertape reader. Each of the five common four-letter nouns in any one pentad started with a different first letter, and no two words in a pentad were similar in meaning or pronounciation. When a pentad was presented twice on a trial, both presentations were in the same left-to-right order.

Design. Each subject went through 48 Brown-Peterson trials: 32 double-presentation trials and 16 single-presentation trials. The 32 double-presentation trials represented two replications of the within-subjects factorial combination of two rehearsal modes on the first presentation, two rehearsal modes on the second presentation, two spacing intervals ( 2 and $12 \mathrm{sec}$ ), and two retention intervals (6 and $18 \mathrm{sec})$. The 16 single-presentation trials comprised four replications of each combination of rehearsal type (primary and secondary) and retention interval ( 2 and $12 \mathrm{sec}$ ).

Procedure. Shown in Figure 1 are outlines of single- and double-presentation trials with the corresponding times used in Experiment I. Each row of the figure represents what appeared in the window of the memory drum.

At the onset of any given trial the subject did not know whether one or two presentations would occur. Following the word ready, a rehearsal cue (highlighted in a distinctive color) was presented. Half the subjects were instructed to engage in primary rehearsal following one cue and secondary rehearsal following the other. The cue-rehearsal mode relationship was reversed for the remaining subjects. After the allotted rehearsal period, the subjects were presented a string of digits grouped into sets of two or three, and during the time available 


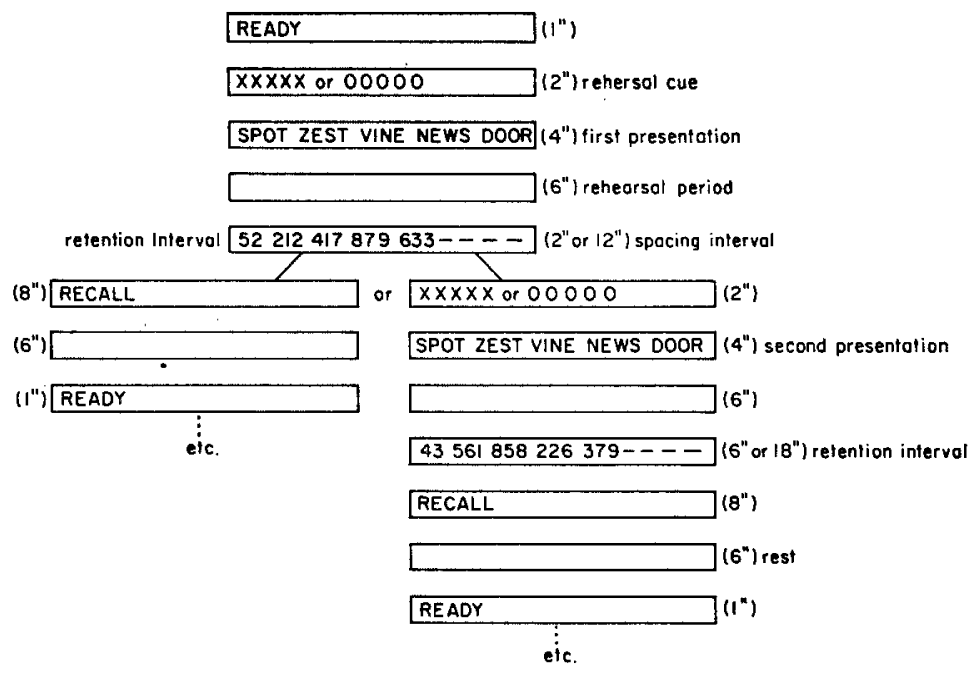

FIG. 1. Outline of single-presentation (on left) and double-presentation (on right) trials in which each frame represents what was seen in the window of a high-speed memory drum. The time intervals used in Experiment I are in parentheses.

they added together the numbers in a pair or triplet, called out the sum, and then classified the sum as odd or even. The first adding-classifying period of a trial was the retention interval for once-presented items and the spacing interval for the twice-presented items. Recall on single-presentation trials followed the first adding-classifying period, while recall on double-presentation trials was after the adding and classifying that followed the second presentation.

Each subject saw the same sets of five words in the same order. Counterbalancing assured that each pentad occurred in every doublepresentation condition or in every single-presentation condition equally often. Pentads were only partially balanced across single- and double-presentation conditions.

All subjects received both primary and secondary rehearsal instructions. These instructions were very detailed, and in the case of secondary rehearsal included several examples of how to form meaningful connections among the five words in a set (specific examples using five three-letter nouns showed how to look for associations, orthographic similarities, pictorial images, and how to generate sentences incorporating the words). On primary rehearsal trials the subjects were instructed to act as if they were attempting to remember a new telephone number; that is, they were told to say the words over and over, either out loud or to themselves, during the entire time available. Speed-accuracy instructions were given for the distractor task and the subjects were aware that their performance on the distractor task was being monitored by the experimenter. Free recall of each pentad was permitted. Each subject received four practice trials to insure that the instructions were understood.

Following the 48 trials, half the subjects were given a written free recall test on which they were asked to write down as many of the words as they could in any order they wished. The other subjects were given a word recognition test which involved half the words from each condition intermixed with an equal number of new four-letter nouns on a sheet of paper. They were instructed to circle the words they had seen in the experiment. Following final free recall or word recognition, all subjects were given a set recognition test. This test involved circling yes or no to groups of two or three words according to whether they did or 
did not occur in the same set of five during the first part of the experiment. All groups of words involved in the set recognition test came from the experiment: Half the groups (the targets) remained intact from the short-term retention trials, while the foils came from different sets and were placed into test groups haphazardly. The 48 groups of words on the test included 24 targets and 24 foils. Two forms of the set recognition test were devised such that the target items on one form comprised the foils on the other. Each form was given to half the subjects. Subjects were allowed as much time as necessary to complete each of the long-term retention tests.

\section{Method: Experiment II}

Subjects. Thirty-six undergraduates at the University of Michigan were paid $\$ 2.00$ for their participation.

Apparatus and materials. The apparatus and materials were identical to those used in Experiment I.

Design and procedure. Twelve of the subjects constituted an uncued control group. These subjects were left to their own devices as far as any rehearsal strategy they might choose to employ. They went through exactly the same sequence of trials as the instructed group, but the uncued group was merely urged to do their best and were told nothing about different kinds of rehearsal strategies.

The remaining 24 subjects constituted the experimental or instructed group. For these subjects, the sequence of events on a given trial was highly similar to that shown in Figure 1 with the following important exceptions: (1) each of 24 subjects saw 36 rather than 48 pentads; (2) the 24 double-presentation trials represented four replications of the within-subjects factorial combination of three spacing intervals $(0,4$, and $18 \mathrm{sec})$ and the primary and secondary rehearsal modes; (3) rehearsal mode was the same for both presentations of any one repeated pentad; (4) the retention interval was always $18 \mathrm{sec}$ on double-presentation trials; (5) the 12 single-presentation trials included two replications of each combination of two rehearsal types and three retention intervals $(0,4$, and $18 \mathrm{sec})$; (6) colored $X s$ and Os were not used as rehearsal cues, rather the word ready and the words themselves were distinctly colored to indicate the type of rehearsal for that item; (7) the presentation time for each pentad was $6 \mathrm{sec}$, and there was no unfilled rehearsal period following presentation; (8) all subjects were administered tests of final free recall, word recognition, and set recognition (in that order); (9) the set recognition test involved four words from each pentad with the first and last words used as foils and the second and fourth used as targets: (10) the middle word from each pentad was used in the word recognition test.

\section{Results and Discussion}

Recall of once-presented items. The initial recall of once-presented items is shown in Figure 2. The scores plotted are the mean proportions of individual words recalled in the single-presentation conditions of Experiments I and II. As is typically the case, recall decreased as length of the retention interval increased, $F(1,15)=94.43, p<.001$, and $F(2$, $46)=46.69, p<.001$, for Experiments I and II, respectively. (These and the following statistical tests do not include the uncued control group unless specified otherwise.) The overall level of recall was higher under secondary than primary instructions in both Experiment I, $F(1,15)=19.62, p<.001$, and Experiment II, $F(1,23)=15.15, p<.001$. The superiority of secondary over primary is limited to the longest retention intervals $F(1,15)=30.49, p<.001$, and $F(2,46)=8.20, p<.005$, for the Rehearsal Instructions $\times$ Retention Interval interaction in Experiments I and II, respectively.

The initial recall results shown in Figure 2 agree with some of our unpublished data in which it was found that levels of initial recall following no distractor activity were somewhat higher under primary than secondary rehearsal, similar to the levels of recall at the 0sec retention interval in Experiment II. 


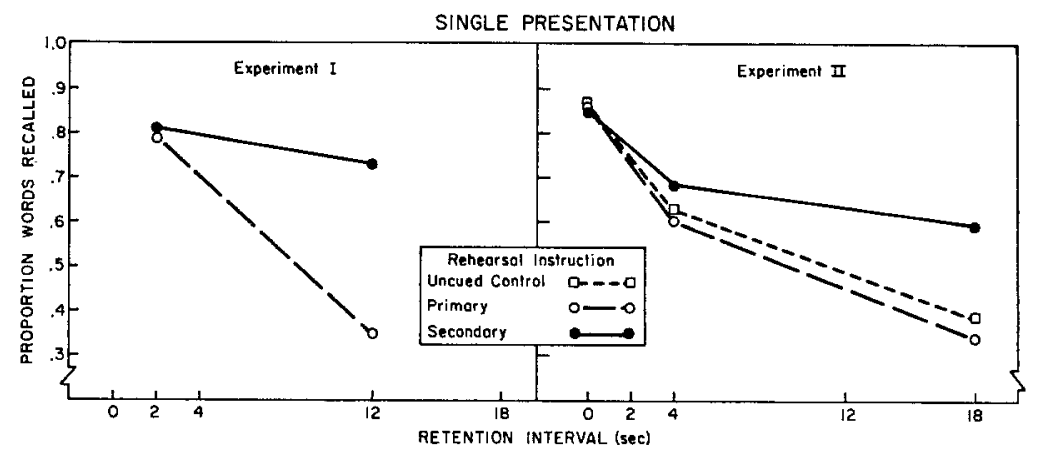

FIG. 2. Proportion of words initially recalled as a function of retention interval in the single-presentation conditions of Experiments I and II.

However, performance after the inclusion of distractor activity, particularly a large amount of such activity, reveals that once-presented items receive much more durable encoding when they are rehearsed in a secondary fashion than when they are subjected to primary rehearsal.

The close correspondence in the levels of recall in the primary and uncued control conditions should be noted. A reasonable interpretation of this finding is that when subjects are free to rehearse in an idiosyncratic fashion in the Brown-Peterson situation, they typically engage in rote (i.e., primary) rehearsal. Uncued subjects rehearse once-presented items in a primary fashion even though it is to their benefit, in terms of level of recall, to use some form of secondary rehearsal.

The proportion correct final free recall, word recognition, and set recognition of the once-presented items are shown in Table 1. These data have been summed across the different retention intervals; the effect of initial retention interval on final recall or recognition was noisy, unsystematic, and contaminated by differences in the level of initial recall. While definite statements about the effects of different types of rehearsal on long-term retention must be tempered by the fact of different levels of immediate recall in some conditions, it does appear that secondary rehearsal yields a more durable memory than does primary rehearsal. The similar levels of final retention performance following primary rehearsal and uninstructed rehearsal suggest that the subjects in these two conditions were processing the items in similar ways.

Recall of repeated items. The proportions of words recalled initially in the double-presentation conditions of Experiment $I$ appear in

TABLE 1

Proportion Correct Final Retentron of ONCE-Presented Items In ExPeriments I and II

\begin{tabular}{|c|c|c|c|c|}
\hline Experiment & $\begin{array}{l}\text { Rehearsal } \\
\text { instructions }\end{array}$ & $\begin{array}{c}\text { Final } \\
\text { free recall }\end{array}$ & $\begin{array}{l}\text { Word } \\
\text { recognition }\end{array}$ & $\begin{array}{c}\text { Set } \\
\text { recognition }\end{array}$ \\
\hline \multirow[t]{2}{*}{ I } & Primary & .04 & .38 & .34 \\
\hline & Secondary & .17 & .56 & .60 \\
\hline \multirow[t]{3}{*}{ II } & Primary & .07 & .40 & .48 \\
\hline & Uncued Control & .10 & .47 & .42 \\
\hline & Secondary & .12 & .68 & .56 \\
\hline
\end{tabular}


Figure 3. The rehearsal instruction in effect on each presentation is noted at the top of each panel. As expected, recall was lower following an 18-sec retention interval than was retention following a 6 -sec retention interval, $F(1,15)=$ $16.52, p<.005$. More items were recalled when the first presentation received secondary rehearsal than when it received primary rehearsal, $F(1,15)=10.72, p<.01$, but rehearsal mode of the second presentation had no overall effect on the level of recall, $F(1,15)=2.90$, primarily because there was an interaction of second presentation rehearsal mode and retention interval, $F(1,15)=7.13, p<.025$. Congruent with the single-presentation results, recall after secondary rehearsal was substantially better than recall after primary rehearsal only at the longer retention interval. This was the only significant interaction in the analysis of double-presentation recall in Experiment $I$.

As shown in Figure 3, there was not a very dramatic spacing effect in Experiment $I$; nevertheless, there was a main effect of spacing interval, $F(1,15)=10.69, p<.01$. Contrary to expectation, multiple comparisons revealed that recall was reliably higher after the long than short spacing interval only when both presentations received primary rehearsal (the

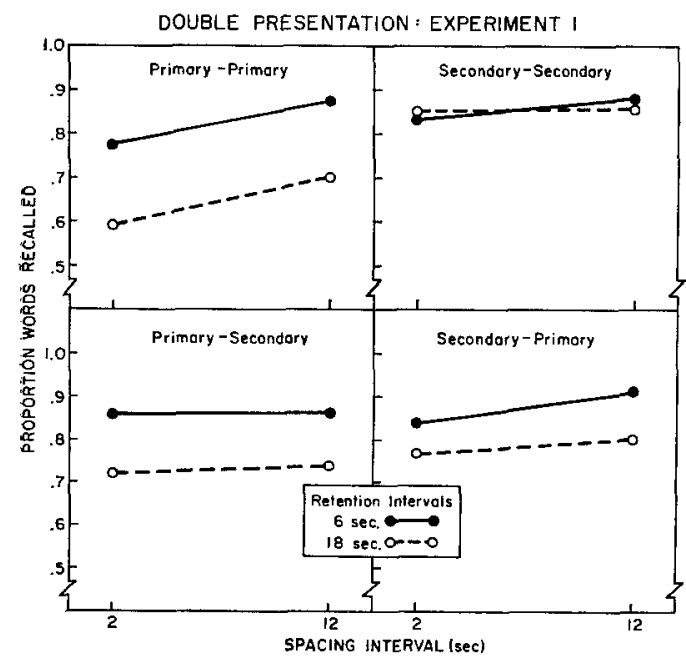

FIG. 3. Proportion of words initially recalled as a function of spacing interval in the double-presentation conditions of Experiment $\mathbf{I}$.

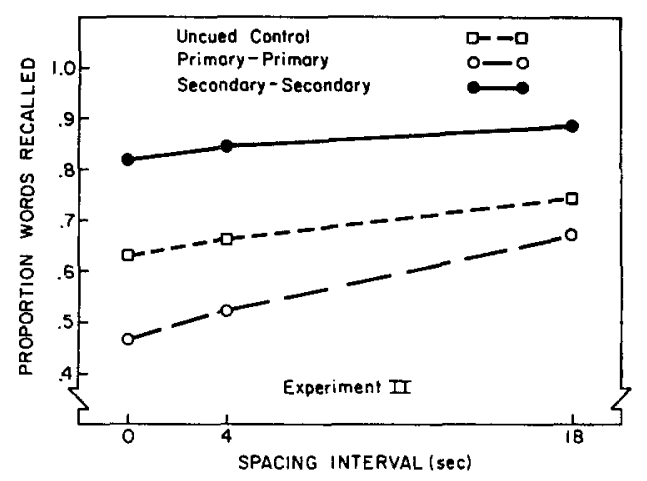

FIG. 4. Proportion of words initially recalled as a function of spacing interval in the double-presentation conditions of Experiment II.

Primary-Primary condition). It was predicted that a spacing effect would not occur in the Primary-Primary case or Secondary-Primary condition but might occur when secondary rehearsal instructions were in effect for the second presentation. In general, the results are directly contrary to these expectations.

Proportions of repeated words recalled initially in the double-presentation conditions of Experiment II are shown in Figure 4. As was true for the once-presented items, recall in the secondary conditions is much higher than recall in the primary conditions, $F(1,23)=$ $74.89, p<.001$. Note that in contrast to the recall of once-presented items, there were more repeated items recalled by the uncued control subjects than by subjects operating under primary rehearsal.

Again, directly contrary to expectation, it is apparent that in Experiment II a more substantial spacing effect occurred in the primary rehearsal condition than in the secondary rehearsal condition, $F(2,46)=3.94, p<.05$, for the Rehearsal Instruction $\times$ Spacing Interval interaction. Not only are the data of the instructed subjects counter to the processing hypothesis, it also appears that the spacing effect for the uncued control subjects fails to coincide with predictions. A reasonable expectation would be that uninstructed subjects should yield a larger spacing effect than either primary or secondary conditions. 
TABLE 2

Proportion Correct Final Retention of Repeated Items In ExPeriments I AND II

\begin{tabular}{clccc}
\hline Experiment & \multicolumn{1}{c}{$\begin{array}{c}\text { Rehearsal } \\
\text { instructions }\end{array}$} & $\begin{array}{c}\text { Final } \\
\text { free recall }\end{array}$ & $\begin{array}{c}\text { Word } \\
\text { recognition }\end{array}$ & $\begin{array}{c}\text { Set } \\
\text { recognition }\end{array}$ \\
\hline \multirow{2}{*}{ I } & Primary-Primary & .06 & .43 & .56 \\
& Secondary-Secondary & .16 & .54 & .78 \\
& Primary-Secondary & .09 & .48 & .73 \\
& Secondary-Primary & .08 & .49 & .69 \\
& Uncued Control & .20 & .69 & .52 \\
& Primary-Primary & .10 & .55 & .47 \\
& Secondary-Secondary & .26 & .79 & .68 \\
& & & & \\
\hline
\end{tabular}

Shown in Table 2 are the final retention scores for the repeated items in Experiments I and II. Two aspects of these data are worth noting. First, the results of Experiment I indicate an incremental effect of secondary processing: Recall and recognition are best when both presentations receive secondary rehearsal, next best when one presentation receives secondary rehearsal, and worst when neither presentation receives secondary rehearsal. Second, it appears that some secondary rehearsal was undertaken by the uncued subjects when they processed the twice-presented items; their repeated-item retention is more like the retention following secondary rehearsal than was the case for the long-term retention of once-presented items.

Other analyses. The frequency with which different kinds of intrusion errors occurred under primary and secondary instructions provides evidence for the maintenance nature of primary rehearsal and the recoding characteristic of secondary rehearsal. Intrusion errors were classified as acoustic, meaningful, or other with regard to the item set on which the intrusion occurred. The mean proportions of acoustic intrusions summed across both studies were .34 on primary trials, .19 on secondary trials, and .20 for the uncued group (these figures are derived from both double- and single-presentation trials and include only the Secondary-Secondary and Primary-Primary conditions from the double presentation con- ditions of Experiment l). The proportions of meaningful intrusions were $.03, .16$, and .07 for primary, secondary, and control instructions, respectively. If a subject commits an intrusion error after primary processing, the intrusion is much more likely to be acoustically than meaningfully similar to the correct response. When operating under secondary instructions, on the other hand, acoustic intrusions occur much less frequently than in the primary case, and secondary rehearsal yields over five times more meaningful intrusions than does primary rehearsal. Primary rehearsal involves processing of the item set at a rather primitive level; whereas, secondary rehearsal results in a recoding that frequently includes a meaningful elaboration of the item.

Further evidence for different encoding under the two rehearsal instructions is provided by final free-recall organization scores from the double-presentation conditions of Experiment II. Organization scores based on the adjacent recall of the words from a particular short-term retention trial were computed using a measure, developed by Bousfield and Bousfield (1966), derived from the differences between the observed item clustering and the item clustering expected by chance. The results of this analysis reveal that over five times as much clustering by item set occurred under secondary rehearsal $(\bar{X}=1.23)$ than under primary rehearsal $(\bar{X}=.24)$. Thus, secondary rehearsal is much more likely to 
yield interrelationships among the to-be-remembered items than is primary rehearsal. Such a result is completely consistent with the idea that secondary rehearsal is an elaborative, integrative process while primary rehearsal is a maintenance scheme.

The order in which words in a given pentad were recalled reveals another difference in performance resulting from primary and secondary rehearsal. In the output analysis a pentad was scored as being in the correct relative output order if the order of output followed the input order, disregarding omissions and intrusions. The mean proportions of pentads with correct relative order of output summed across both studies were .52 on primary trials, .34 on secondary trials, and .38 for the uncued group (these figures include both single and double presentation trials and were derived from just the Secondary-Secondary and Primary-Primary conditions of the double-presentation conditions of Experiment I). Thus, recall order following primary rehearsal is much more likely to be the same as input order than is the order after secondary processing. It would appear that the integration and elaboration involved in secondary processing is less likely to preserve the order of input than is the rote, cyclic activity that occurs during primary rehearsal.

It might be possible to argue that the failure to obtain a spacing effect under secondary rehearsal results from a ceiling effect on recall. Consider the results of Experiment II shown in Figure 4. Under secondary rehearsal at an interval of zero, the subjects are recalling slightly more than four of the five words in a set, and at a spacing of 18 seconds about 4.5 of the five words are recalled under secondary rehearsal. The possibility of a performance ceiling seems unlikely upon consideration of the recall of subjects who performed above and below the median level of recall (the following conclusions are the same when median performance is based on either the recall level of once-presented items or on the recall level of repeated ones). Regardless of rehearsal in- structions, above and below median subjects yielded spacing functions highly similar in shape to those shown in Figure 4. Below median performance under secondary rehearsal ranges from 3.8 to 4.2 items across lags.

Examination of correct additions and classifications in the spacing and retention intervals revealed approximately the same amount of distractor activity in the instructed and uncued conditions. The slight superiority of distractor performance when the to-be-remembered items were rehearsed in a secondary fashion rules out the possibility that the higher level of initial recall in the secondary conditions resulted from less attention to the distractor task. In addition, the distractor results are consistent with the idea that after the initial encoding effort, secondary rehearsal requires less processing capacity than does primary rehearsal (cf. (Griffith \& Johnston, 1973)).

\section{EXPERIMENT III}

A third experiment was designed to test directly the possibility that secondary processing of the second presentation is a crucial factor in the spacing effect. The first (or only) presentation of items in all conditions was limited to four seconds in which the subjects were required to read the pentad out loud. There was no blank rehearsal period after the first (or only) presentation. Cues prior to the second presentation required either primary or secondary rehearsal, and the presentation and rehearsal times for the second presentation were the same as those used in Experiment I (see Figure 1). It was felt that the shorter total presentation time and the reading requirement would guarantee that the first presentation always received primary rehearsal. Thus, any differences in the spacing effect would result from the kind of rehearsal given the second presentation.

\section{Method}

Subjects. Twelve undergraduate students at the University of Michigan were paid $\$ 2.00$ for their participation in this experiment. 
Apparatus and materials. The apparatus and materials were the same as those used in Experiments I and II.

Design and procedure. The design may be considered as a replication of conditions Primary-Primary and Primary-Secondary that were examined in Experiment I. Each subject received 33 Brown-Peterson trials: 12 Primary-Primary trials; 12 Primary-Secondary trials; and 3 single-presentation trials at each of three retention intervals $(0,4$, and $18 \mathrm{sec})$. For the repeated items the spacing intervals were 0,4 , and $18 \mathrm{sec}$, and the retention interval was $18 \mathrm{sec}$. The first or only presentation was uncued and the subjects were required to read the pentad outloud during the 4 sec it appeared in the window of the memory drum. The presentation times and cuing procedures for the second presentation were exactly the same as in Experiment I (see Figure 1). The remainder of the procedure was also identical to that used in Experiment $\mathrm{I}$.

\section{Results and Discussion}

Initial recall of once-presented items. The proportions of once-presented words initially recalled in Experiment III are shown in Figure 5. As the length of the retention interval in-

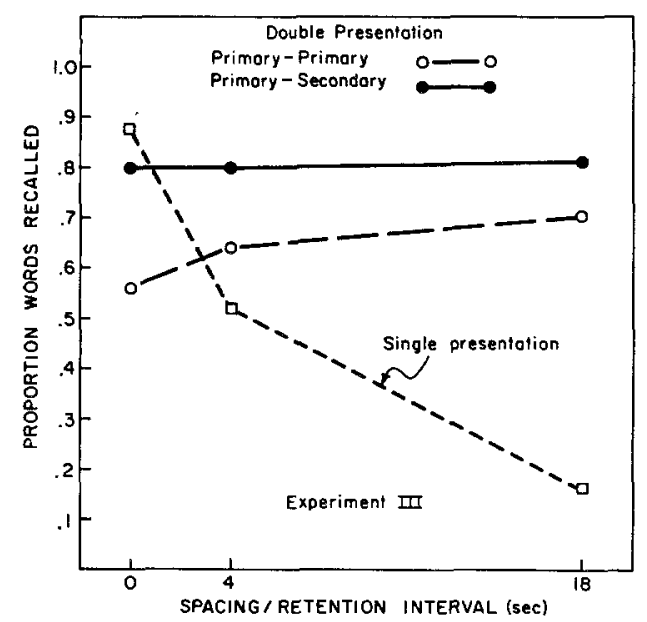

FIG. 5. Proportion of words initially recalled as a function of spacing interval (double-presentation conditions) or retention interval (single-presentation condition) of Experiment III. creased, there was a dramatic decrease in initial recall, $F(2,22)=74.13, p<.001$. It is interesting to note that initial recall after 18 seconds (.16) is less than half that observed (.34) in the primary rehearsal conditions of Experiment II (see Figure 2). This difference may be attributed to the reading requirement and lack of a rehearsal period following the first presentations in Experiment III.

Initial recall of repeated items. The proportions of repeated words recalled initially are also shown in Figure 5. Obviously, there was no spacing effect when the second presentation received secondary rehearsal, $F<1$, but there was a large spacing effect when the second presentation received primary processing, $F(2$, $22)=3.51, p<.05$. Once again, contrary to expectation, primary rehearsal of the second presentation yields a spacing effect.

The results of the final retention tests are consistent with those of Experiments I and II; they add little, and will not be reported here.

\section{General Discussion}

The results obtained in the present experiments are completely consistent and unambiguous in their empirical form, if not in their implications. As for the overall consequences of primary and secondary processing, our expectations were completely supported. However, as for the influence of type of processing on the spacing effect, our expectations were completely violated. Both of these matters merit additional comment.

\section{Types of Rehearsal}

The distinction between rehearsal as a rote maintenance activity and rehearsal as a constructive, elaborative activity is clearly both viable and important. The complete pattern of differential consequence of the two types of rehearsal in the present results is quite remarkable. In initial recall, there is a very strong interaction of type of rehearsal with retention interval: At long retention intervals 
(or in final retention), secondary processing produces much better recall than does primary rehearsal, but at short retention intervals, primary rehearsal results in performance as good as or, as some of our unpublished data reveal, much better than performance resulting from secondary rehearsal. The pattern of intrusions in initial recall differs as well: Secondary processing tends to result in semantic intrusions, whereas primary processing leads to acoustic intrusions. Another difference that is quite apparent in the data is that words recalled from a pentad given primary rehearsal are very likely to be output in an order that matches their input order, even though correct order was not required during initial recall. On the other hand, the words recalled from a pentad given secondary rehearsal were seldom output in an order corresponding to their input order. Primary processing is a rote, cyclic activity that preserves the input order of the rehearsed set; secondary processing involves idiosyncratic integration that reorders the items in a set. A final difference is that secondary rehearsal produced a greater level of clustering by input pentad during final recall than did primary rehearsal. Once again, that result is completely consistent with the idea that secondary processing is more of an integrative activity than is primary processing.

\section{Level of Processing and the Spacing Effect}

The present results provide little, if any, support for the notion that the spacing effect is attributable to a change in level of processing of a second presentation as the spacing of that presentation from a first is increased. In fact the strong spacing effects obtained in those conditions where primary processing of the second presentation was required at all spacing intervals run directly contrary to the predictions of the level of processing view. The present results also pose some problems for the encoding variability explanation of the spacing effect. In particular, the lack of any substantial spacing effect in the Secondary-Secondary conditions seems inconsistent with the appa- rent predictions derived from encoding-variability theory.

There is, however, an elaborated version of the encoding variability theory that seems consistent with the present results. The necessary assumptions are the following.

(a) During or prior to the primary rehearsal of a given pentad, there is a certain amount of obligatory secondary or semantic processing (cf. (Craik \& Lockhart, 1972; Miller, 1962)). The individual words are certainly processed to a semantic level when they are first attended to even though it may be their acoustic representation that is cycled in a rote fashion during their subsequent rehearsal. It seems plausible that the formation of certain idiosyncratic or not so idiosyncratic associations between some members of a pentad is automatic and unavoidable. Some support for this assumption is provided by the final recall results in Tables 1 and 2. In those tables, final recall ranges between about one-fourth and one-half the level of final recall of items given secondary rehearsal. It is highly unlikely that it is the acoustic representation of the primary-processed items that provides the basis for their final recall.

(b) On the average, the secondary encoding that does occur on primary-rehearsal trials is relatively unstable. Any such encodings would typically consist of an association between only some fraction of the five words in a pentad (perhaps two), and since no substantial integration had been achieved, that encoding would become unavailable relatively rapidly during the context change effected by distractor activity during the spacing interval. Recall level would be poor after any appreciable amount of such distractor activity, and any obligatory secondary processing that occurred during a second presentation would be relatively independent of the initial encoding. Thus, primary processing constitutes an encoding condition highly susceptible to increasing encoding variability with increasing spacing. It is that encoding instability or variability that produced both the poor recall 
of once-presented items in the present studies and the substantial spacing effects in the recall of repeated items.

(c) During the secondary processing of a typical pentad, however, a relatively stable encoding is achieved. Such integrative encodings tend to remain available even over long periods of distractor activity. Since performance declines slowly following a single presentation, the secondary encoding at the time of a second presentation tends to be highly correlated with the initial encoding, which means that even after considerable spacing intervals there is marginal encoding variability and little in the way of a spacing effect.

One implication of these assumptions is that the recall advantage of repeated over nonrepeated items will be less for items that receive secondary rehearsal than in the case where primary rehearsal instructions are in effect. This implication is clearly supported by the results of Experiment II (see Figures 2 and 4). In the case where once- and twice-presented items have the same retention interval (18 sec) and there is maximum spacing for the repeated items, the difference between repeated and nonrepeated recall is .29 for secondary rehearsal and .39 for primary rehearsal. This difference represents a half-item advantage for primary over secondary.

The foregoing amalgamation of encodingvariability theory and the levels of processing view is admittedly post factum, but it does seem to hold considerable promise. Fortunately, this interactive theory is testable, because it is easy to conceive of experiments that orthogonally vary both type of rehearsal and the amount of encoding. Such experiments should provide a reasonable way of determining the independent and joint effects of encoding and rehearsal in retention.

\section{REFERENCES}

Atkinson, R. C., \& Shiffrin, R. M. Human memory: A proposed system and its control processes. In K. W. Spence \& J. T. Spence (Eds.), The psychology of learning and motivation. Vol. 2. New York: Academic Press, 1968. Pp. 89-195.

BJork, R. A. Repetition and rehearsal mechanisms in models of short-term memory. In D. A. Norman (Ed.), Models of human memory. New York: Academic Press, 1970. Pp. 307-330.

Bousfield, A. K., \& Bousfield, W. A. Measurement of clustering and of sequential constancies in repeated free recall. Psychological Reports, 1966, 19, 935-942.

CRAIK, F. I. M., \& LockHART, R. S. Levels of processing: A framework for memory research. Journal of Verbal Learning and Verbal Behavior, 1972, 11, 671-684.

CRAIK, F. I. M., \& WatkINS, M. J. The role of rehearsal in short-term memory. Journal of Verbal Learning and Verbal Behavior 1973 12, 599-607.

De Remer, P., \& D'Agostino, P. R. Locus of distributed lag effect in free recall. Journal of Verbal Learning and Verbal Behavior, 1974, 13, 167-171.

EsTES, W. K. Statistical theory of distributional phenomena in learning. Psychological Review, 1955, 62, 369-377. (a)

EsTES, W. K. Statistical theory of spontaneous recovery and regression. Psychological Review, 1955, 62, 145-154. (b).

GreENo, J. G. Conservation of information processing capacity in paired-associate memorizing. Journal of Verbal Learning and Verbal Behavior, 1970, 9, 581-586.

Griffith, D., \& Johnston, W. A. An informationprocessing analysis of visual imagery. Journal of Experimental Psychology, 1973, 100, 141-146.

HinTZMAN, D. L. Theoretical implications of the spacing effect. In R. L. Solso (Ed.), Theories in cognitive psychology: The Loyola symposium. Potomac, Maryland: Lawrence Erlbaum Associates, 1974.

JACOBY, L. L. Encoding processes, rehearsal, and recall requirements. Journal of Verbal Learning and Verbal Behavior, 1973, 12, 302-310.

JACOBY, L. L., \& BARTZ, W. H. Rehearsal and transfer to LTM. Journal of Verbal Learning and Verbal Behavior, 1972, 11, 561-565.

MADIGAN, S. A. Intraserial repetition and coding processes in free recall. Journal of Verbal Learning and Verbal Behavior, 1969, 8, 828-835.

Martin, E. Stimulus meaningfulness and pairedassociate transfer: An encoding variability hypothesis. Psychological Review, 1968, 75, 421-441.

Melton, A.W.The situation with respect to the spacing of repetitions and memory. Journal of Verbal Learning and Verbal Behavior, 1970, 9, 596-606.

Meunier, G. F., Ritz, D., \& MeUnier, J. A. Rehearsal of individual items in short-term memory. Journal of Experimental Psychology, 1972, 95, 465-467. 
Miller, G. A. Decision units in the perception of speech. IRE Transactions on Information Theory, 1962, IT-8, 81-83.

UNDERWOOD, B. J. A breakdown of the total-time law in free-recall learning. Journal of Verbal Learning and Verbal Behavior, 1970, 9, 573-580.
Woodward, A. E., BJork, R. A., \& Jongeward, R. H. Recall and recognition as a function of primary rehearsal. Journal of Verbal Learning and Verbal Behavior, 1973, 12, 608-617.

(Received June 24, 1974) 